\title{
An elastoplastic 1D Winkler model for suction caisson foundations under combined loading
}

\author{
S.K. Suryasentana, B.W. Byrne \& H.J. Burd \\ University of Oxford, UK
}

A. Shonberg

Ørsted Wind Power, London, UK

\begin{abstract}
Most existing Winkler models use non-linear elastic soil reactions to capture the non-linear behaviour of foundations. These models cannot easily capture phenomena such as permanent displacement, hysteresis and the influence of combined loading on the failure states. To resolve these shortcomings, an elastoplastic Winkler model for suction caisson foundations under combined loading is presented. The proposed model combines Winkler-type linear elastic soil reactions with local plastic yield surfaces to model the nonlinear soil response using standard plasticity theory, albeit in a simplified one-dimensional (1D) framework. The results demonstrate that the model reproduces the appropriate foundation behaviour, comparing closely to three-dimensional finite element (3DFE) analyses but with the advantage of rapid computation time.
\end{abstract}

\section{INTRODUCTION}

\subsection{General Background}

Winkler models are widely used to design deep foundations such as piles. However, in recent work (Gerolymos and Gazetas, 2006; Varun et al., 2009; Suryasentana et al., 2017), Winkler models have also been developed for shallow foundations such as caisson foundations. While these design methods may not be as accurate as more rigorous approaches such as the three-dimensional finite element (3DFE) method, Winkler models have the advantages of being relatively fast and easy to use.

Winkler models simplify the three-dimensional (3D) foundation-soil interaction problem into a more tractable one-dimensional (1D) problem, with the foundation replaced by a beam and the soil continuum by Winkler 'springs' (also termed as soil reactions in this paper). To simulate the non-linear response of soil, the Winkler models adopt non-linear elastic soil reactions. Examples of such models include the $p-y$ and $t-z$ methods (API, 2010; DNV, 2014) used to design laterally and axially loaded piles respectively.

Nevertheless, there are shortcomings with these existing non-linear Winkler models. For example, the non-linear elastic soil reactions used in the $p-y$ or $t-z$ methods for piles cannot reproduce observed cyclic loading phenomena such as permanent displacement or hysteresis. Moreover, they cannot account for combined loading effects on the failure state.

\subsection{Proposed Model}

Recently, Suryasentana et al. (2017) developed a 1D Winkler model, calibrated against 3DFE analyses, to accurately predict suction caisson behaviour in linear elastic soil for six degrees of freedom (dof) loading. However, this model can only be applied to loading conditions where the soil response can be approximated as linear elastic.

This paper extends the 1D Winkler model developed in Suryasentana et al. (2017) to allow predictions of non-linear caisson behaviour in undrained clay under combined planar vertical $V$, horizontal $H$ and moment $M$ loading. The extension involves coupling linear elastic soil reactions with local plastic yield surfaces, which are calibrated against rigorous 3DFE failure state analyses.

The governing mechanics of the proposed 1D model is based on the same elastoplasticity framework used in 3DFE analyses. This allows straightforward reproduction of the 3DFE predictions, but with higher efficiency due to dimensionality reduction. Consequently, the proposed 1D model allows fast and accurate solutions of caisson behaviour in elastoplastic soil for design assessment under fatigue, serviceability and ultimate limit states (FLS, SLS, ULS). This enables an efficient design process, with the 1D model used to quickly shortlist potential designs from a large candidate space, before further refinement is conducted with 3DFE analyses. 


\section{METHODS}

\subsection{D Model}

The 1D model adopted in this paper is similar to that detailed in Suryasentana et al. (2017) and it is briefly described as follows. The 1D model is a simplified representation of the original 3D caisson-soil interaction problem, where the caisson structure and soil continuum are replaced by a $1 \mathrm{D}$ rigid body and Winkler-type soil reactions respectively.

Figure 1 shows a schematic diagram of the original $3 \mathrm{D}$ caisson-soil problem and the $1 \mathrm{D}$ model representation. There are two types of soil reactions in this model: distributed soil reactions that act along the caisson skirt (referred to as the 'skirt soil reactions' and indicated as $\mathrm{h}^{\text {skirt }}, \mathrm{m}^{\text {skirt }}$ and $\mathrm{v}^{\text {skirt }}$ in Figure 1) and concentrated soil reactions that act on the caisson base, including the soil plug (referred to as the 'base soil reactions' and indicated as $\mathrm{h}^{\text {base }}, \mathrm{m}^{\text {base }}, \mathrm{v}^{\text {base }}$ in Figure 1$) . h^{\text {skirt }}, v^{\text {skirt }}$ and $m^{\text {skirt }}$ represent the distributed horizontal force, vertical force and rotational moment along the skirt length, while $h^{\text {base }}, \mathrm{v}^{\text {base }}$ and $\mathrm{m}^{\text {base }}$ represent the concentrated horizontal force, vertical force and rotational moment at the base.

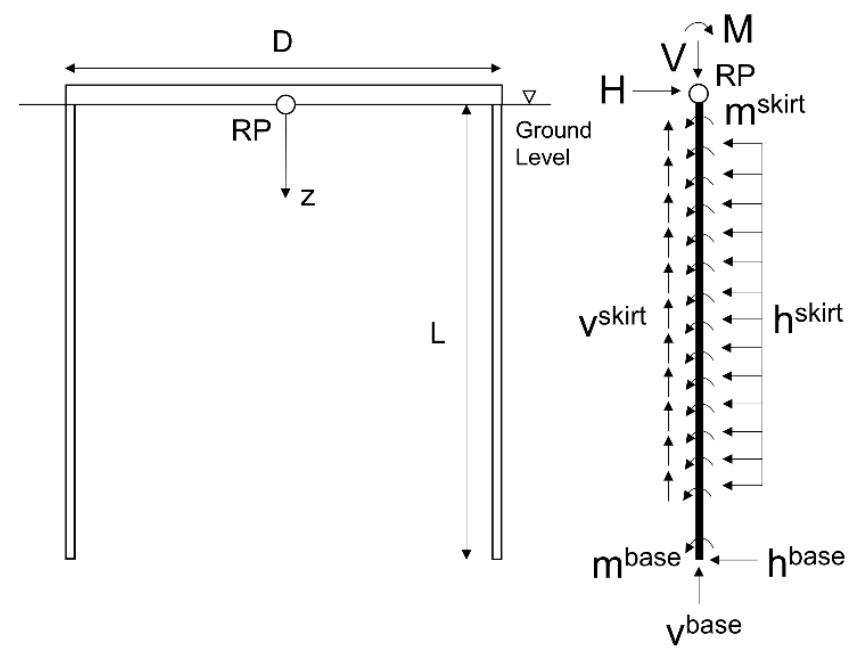

Figure 1. Schematic diagram of an embedded suction caisson foundation (left) and its corresponding simplified 1D representation (right), where RP is the loading reference point. $v, h$ and $m$ are the vertical, horizontal and rotational soil reactions.

There are, however, a few notable differences between the 1D model adopted in this paper and that described in Suryasentana et al. (2017). First, as this paper is only concerned with planar VHM loading, there are only 3 components $(v, h, m)$ for each soil reaction, which correspond to the vertical $w$, horizontal $u$ and rotational $\theta$ degrees of freedom (dof). Second, unlike the linear elastic soil assumed in Suryasentana et al. (2017), the current paper assumes a linear elastic-perfectly plastic soil. This gives an ultimate limit to the soil response, which the previous 1D model was not able to capture. To simulate this behaviour, the 1D model in this paper couples the linear elastic soil reactions with local plastic yield surfaces. These local yield surfaces are a direct analogy of the elemental yield surfaces in the soil reactions space (consisting of $v, h, m$ components). Just as the canonical yield surfaces determine the set of allowable elemental stress states, the local yield surfaces determine the set of allowable soil reaction states.

The mechanics of the coupled soil reactions-yield surfaces model can be explained by standard plasticity theory. For soil reaction states lying inside the local yield surface, the soil response is linear elastic with the incremental response given by:

$\delta \boldsymbol{p}=\boldsymbol{k}_{\boldsymbol{e}} \delta \boldsymbol{u}$

where $\boldsymbol{p}=$ soil reactions $\{v, h, m\}, \boldsymbol{k}_{\boldsymbol{e}}=$ elastic stiffness matrix and $\boldsymbol{u}=$ local displacements $\{w, u, \theta\}$. $\boldsymbol{k}_{\boldsymbol{e}}$ can be obtained from Suryasentana et al. (2017) as the caisson dimensions $(L / D=1)$ and elastic soil properties $(v=0.49)$ adopted in this paper are identical. However, for simplicity and faster numerical convergence, the coupling terms between $h$ and $m$ in $\boldsymbol{k}_{\boldsymbol{e}}$ are ignored (the exclusion of these coupling terms will mainly impact the accuracy of the elastic horizontal and rotational predictions). Thus, $\boldsymbol{k}_{\boldsymbol{e}}$ for the skirt and base soil reactions are as follows:

$$
\begin{aligned}
\boldsymbol{k}_{\boldsymbol{e}}^{\text {skirt }} & =G\left[\begin{array}{ccc}
4.28 & 0 & 0 \\
0 & 6.51 & 0 \\
0 & 0 & 1.17 D^{2}-0.12 z D
\end{array}\right] \\
\boldsymbol{k}_{\boldsymbol{e}}^{\text {base }} & =G D\left[\begin{array}{ccc}
2.4 & 0 & 0 \\
0 & 1.17 & 0 \\
0 & 0 & 0.42 D^{2}
\end{array}\right]
\end{aligned}
$$

where $G=$ shear modulus of soil, $D=$ caisson diameter, $z=$ depth below ground level (see Figure 1).

When the soil reaction states reach the local yield surface, the soil response becomes elastoplastic, with incremental behaviour given by:

$\delta \boldsymbol{p}=\boldsymbol{k}_{\boldsymbol{e p}} \delta \boldsymbol{u}$

where $\mathbf{k}_{e p}=$ elastoplastic stiffness matrix. By convention, the local yield surface $f(\boldsymbol{p})$ is defined as follows: $f<0$ for states inside the yield surface, $f=0$ for states on the yield surface, and $f>0$ for inadmissible states outside the yield surface.

When elastoplastic yielding occurs, permanent plastic displacements accumulate with the total displacement increment $\delta \boldsymbol{u}$ composed of elastic and plastic parts:

$\delta \boldsymbol{u}=\delta \boldsymbol{u}_{\boldsymbol{e}}+\delta \boldsymbol{u}_{\boldsymbol{p}}$

The elastic displacement increment $\delta \boldsymbol{u}_{\mathrm{e}}$ is determined by the soil reaction increment through:

$\delta \boldsymbol{u}_{\boldsymbol{e}}=\boldsymbol{K}_{\boldsymbol{e}}{ }^{-1} \delta \boldsymbol{p}$

The plastic displacement increment $\delta \boldsymbol{u}_{\mathrm{p}}$ is determined by the flow rule: 
$\delta \boldsymbol{u}_{\boldsymbol{p}}=\lambda \frac{\partial g}{\partial \boldsymbol{p}}$

where $g(\boldsymbol{p})$ is a plastic potential function and $\lambda$ is a non-negative, scalar plastic multiplier. When yielding occurs, the incremental soil reaction $\delta p$ must remain on the local yield surface. This is enforced by the consistency condition:

$\left(\frac{\partial f}{\partial \boldsymbol{p}}\right)^{\mathrm{T}} \delta \boldsymbol{p}=0$

Following the conventional approach for linear elastic-perfectly plastic models, kep is obtained from:

$\boldsymbol{k}_{e p}=\boldsymbol{k}_{\boldsymbol{e}}-\frac{\boldsymbol{k}_{\boldsymbol{e}}\left(\frac{\partial g}{\partial p}\right)\left(\frac{\partial f}{\partial p}\right)^{\mathrm{T}} \boldsymbol{k}_{e}}{\left(\frac{\partial f}{\partial p}\right)^{\mathrm{T}} \boldsymbol{k}_{e}\left(\frac{\partial g}{\partial p}\right)}$

For this paper, an associated flow rule is assumed i.e. $g(\boldsymbol{p})=f(\boldsymbol{p})$. The local yield surface $f(\boldsymbol{p})$ is calibrated using the limiting soil reactions extracted from the 3DFE analyses, which is described in Section 2.3.

The 1D model was implemented numerically using the Galerkin finite element methodology, where twonoded 1D soil elements (each with a linear shape function and two Gauss points) representing the skirt soil reactions are tied to two-noded 1D caisson rigid bar elements. The base soil reaction is represented by a lumped model tied to the bottom node of the deepest caisson element. The explicit Runge-Kutta $(4,5)$ algorithm (Dormand and Prince, 1980) was used for the integration process during elastoplastic behaviour and the full Newton-Raphson procedure was used to obtain the system solution.

\subsection{DFE Model}

The 3DFE analyses were carried out using the finite element program ABAQUS v6.13 (Dassault Systèmes 2010). The 3DFE model consists of a suction caisson foundation (of unit diameter $D$ and unit skirt length $L=D$ ) embedded in homogeneous soil, which is similar to that used in Suryasentana et al. (2017).

The mesh domain is set as $8 D$ for the diameter and $6 D$ for the depth, which was verified to be large enough to avoid boundary effects for load capacity predictions. Mesh convergence analyses were also carried out to determine the required mesh fineness. Due to symmetry of the problem, only half of the caisson and soil domain was modelled. A typical mesh of the 3DFE model is shown in Figure 2.

The soil was defined as weightless, homogeneous and linear elastic-perfectly plastic. The soil is assumed to obey the von Mises yield criterion with an associated flow rule. The Young's modulus $E$ of the soil is set as $1000 \sqrt{3} s_{\mathrm{u}}$ (where $s_{\mathrm{u}}$ is the undrained shear strength) and the Poisson's ratio is set as 0.49 .

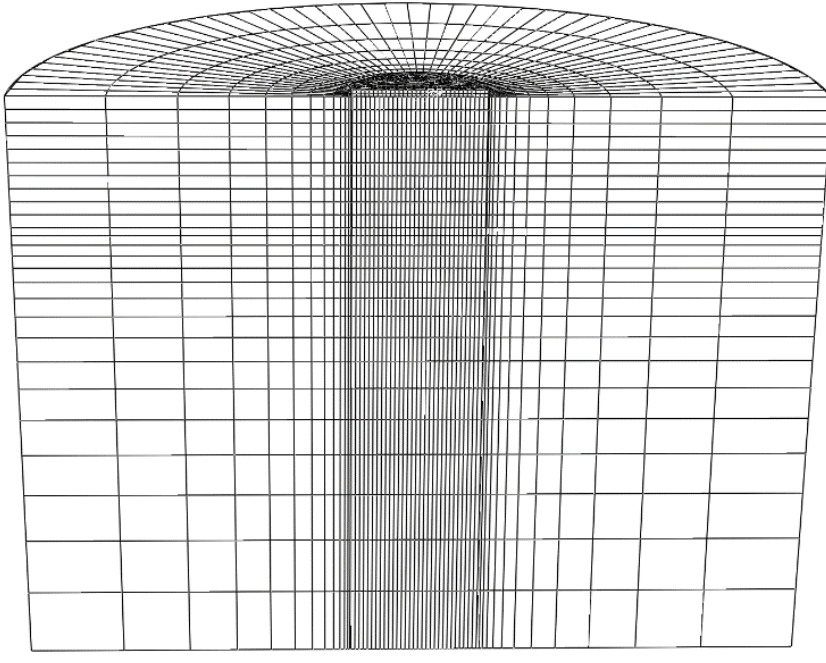

Figure 2. Mesh used for the 3DFE analyses. The diameter and depth of the mesh domain is set as $8 D$ and $6 D$ respectively.

Fully-integrated, linear, brick elements C3D8H were used to model the soil elements. The caisson was modelled as being entirely rigid using rigid body constraints. The caisson reference point was set at RP, as shown in Figure 1. Contact breaking between the caisson and soil was prevented using tie constraints at the caisson-soil interface. Displacements were fixed in all directions at the bottom of the mesh domain and in the radial directions at the periphery.

\subsection{Calibration of local yield surfaces}

To calibrate the local yield surfaces in the proposed 1D model, a series of 3DFE analyses were carried out to obtain the limiting soil reactions. These analyses involved the determination of the global VHM failure envelope of the caisson-soil interaction problem (Bransby and Yun, 2009; Gourvenec and Barnett, 2011; Vulpe, 2015). This was done using mixed load and displacement control, where load control was used in the $V$ load space while displacement control was used in the $H M$ load space. In total, four vertical loads $\left(V / V_{0}=0,0.25,0.5,0.75\right.$ where $V$ is the vertical load applied at RP and $V_{0}$ is the uniaxial vertical load capacity) were applied before displacement probes were applied in the $H M$ load space. This determines the $H M$ failure envelopes at fixed levels of $V$. It is hypothesized that, just as there exists a failure envelope that limits the global load space, there also exists a limiting envelope in the soil reactions space (termed as 'local yield surface' in this paper), which can be identified using the limiting soil reactions extracted from the 3DFE analyses.

The limiting soil reactions were extracted from the 3DFE results at the end of each displacement probe, corresponding to a global failure state of the caissonsoil interaction problem. For simplicity, the limiting skirt soil reactions are assumed to be constant along 
the skirt and are computed as the average of the soil reactions along the skirt.

To represent the local yield surface, an ellipsoid function $f(\boldsymbol{p})$ is adopted:

$f=\left(\frac{v}{v_{0}}\right)^{2}+\left(\frac{h}{h_{0}}\right)^{2}+\left(\frac{m}{m_{0}}\right)^{2}+\alpha\left(\frac{h m}{h_{0} m_{0}}\right)-1$

where $v_{0}, h_{0}$ and $m_{0}$ are the limiting uniaxial vertical, horizontal and moment soil reactions (i.e. the uniaxial capacities in the soil reactions space) and $\alpha$ is a parameter that governs the rotation of the ellipsoid in the $h m$ space. This ellipsoid function was adopted as it has favourable theoretical properties such as global convexity.

The unknown parameters $v_{0}, h_{0}, m_{0}$ and $\alpha$ were identified by running least squares regression against the limiting soil reactions extracted from the 3DFE results. The best-fit parameters for the skirt and base local yield surfaces are shown in Table 1.

Table 1. Best-fit parameters for the skirt and base local yield surfaces

\begin{tabular}{lcl}
\hline Parameter & Skirt & Base \\
\hline$v_{0} / \mathrm{s}_{\mathrm{u}}$ & $A^{\text {skirt }}$ & $9.1 A^{\text {base }}$ \\
$h_{0} / \mathrm{s}_{\mathrm{u}}$ & $2.07 A^{\text {skirt }}$ & $1.34 A^{\text {base }}$ \\
$m_{0} / \mathrm{s}_{\mathrm{u}}$ & $0.19 A^{\text {skirt }} D$ & $0.72 A^{\text {base }} D$ \\
$\alpha$ & -1.23 & -0.47 \\
\hline
\end{tabular}

where $D$ is the caisson diameter, $A^{\text {skirt }}$ (skirt surface area per metre length basis) $=\pi \mathrm{D}$ and $A^{\text {base }}=\pi \mathrm{D}^{2} / 4$

The local yield surface contours generated by Equation 17 and the best-fit parameters in Table 1 are compared against the 3DFE limiting soil reactions in Figure 3. Although the global vertical load $V$ is fixed while the $H M$ failure envelope is probed, the distribution of the vertical load between the skirt and base soil reactions is not constant. Thus, each of the limiting soil reactions is associated with a different $v / v_{0}$ value. To simplify the process, the average of these $v / v_{0}$ values (for each dataset corresponding to a fixed $V$ ) are used in Equation 17 to predict the $\mathrm{hm}$ contours for each $V / V_{0}$; their values are shown in the contour labels in Figure 3. For $V / V_{0}=\{0,0.25,0.5,0.75\}, v / v_{0}=\{0$, $0.1,0.35,0.7\}$ for the skirt soil reactions and $\{0,0.32$, $0.57,0.77\}$ for the base soil reactions.

It was observed that the predicted local yield surface contours are good approximations to the limiting base soil reactions at low vertical loads. However, at higher vertical loads $\left(V / V_{0} \geq 0.5\right)$, there is less agreement as the ellipsoid function cannot capture the change in yield surface geometry. The fit is less than ideal for the limiting skirt soil reactions as they do not conform closely to an ellipsoidal shape.

However, although these simplified local yield surfaces do not match well on a local level, they produce reasonably accurate global predictions (as will be shown in Section 3.2).
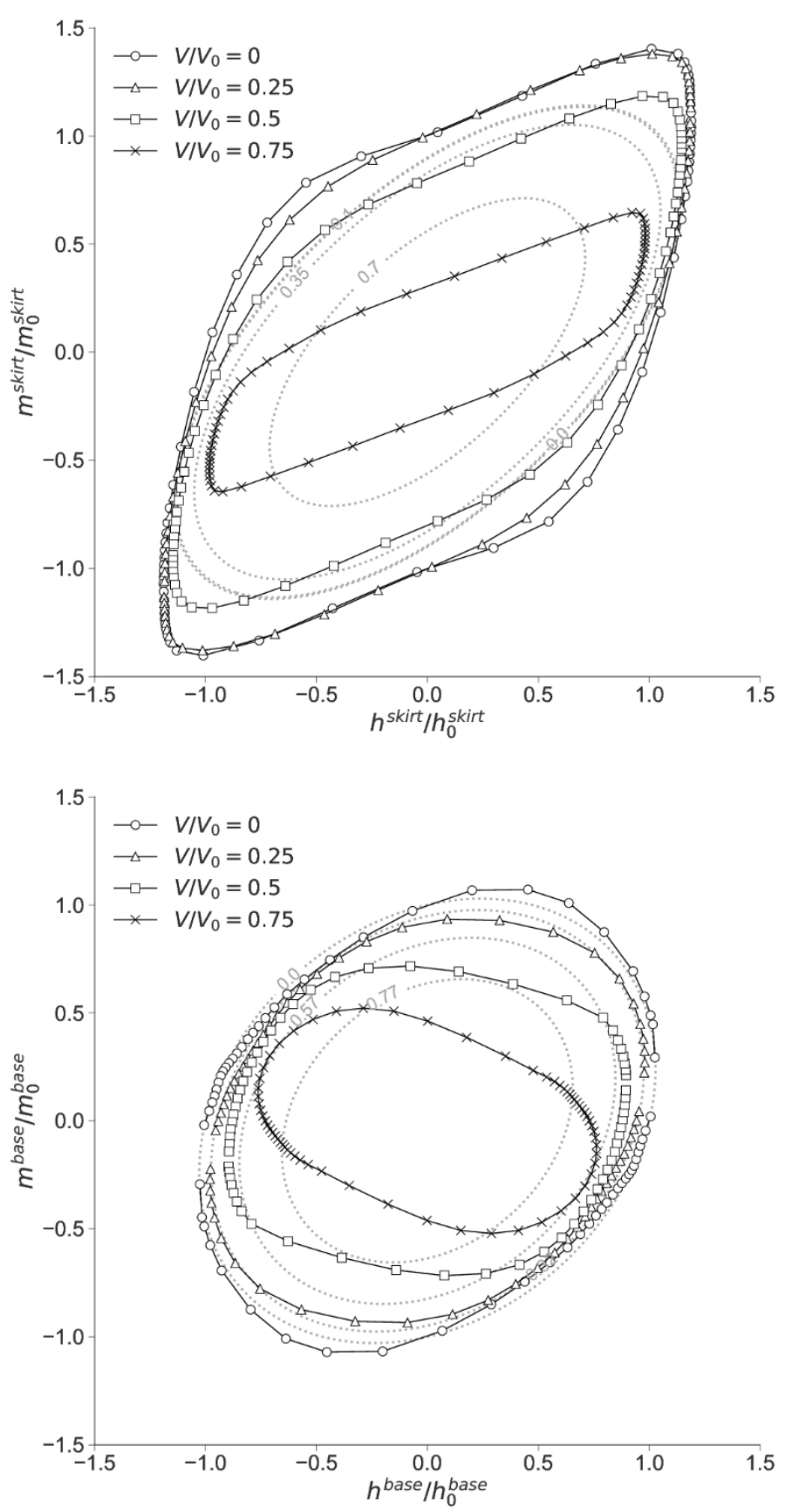

Figure 3. Comparison of the 3DFE limiting skirt and base soil reactions (as depicted by the markers in the figure) against the local yield surface contours (as depicted by the grey dotted lines) predicted by Equation 17 and Table 1 . The average $v / v_{0}$ values (for each dataset corresponding to a fixed $V$ ) are shown in the contour labels and they are used in Equation 17 to produce the contours.

\subsection{Evaluation of Models}

To compare the predictions between the 1D model and the 3DFE model, three types of evaluations were implemented. First, the uniaxial load capacities (which are the load capacities under the application of $V, H$ and $M$ individually) were evaluated to assess the accuracy of the models for simple loading cases. Next, the influence of combined loading on failure states was assessed by using the 1D and 3DFE models to find the failure envelopes of the caisson in the $V H M$ 
load space. Finally, a single cyclic load test was simulated using both models to assess the capability of capturing permanent displacement and hysteresis.

\section{RESULTS}

\subsection{Uniaxial Load Capacities}

Table 2 shows the uniaxial vertical $V_{0}$, horizonal $H_{0}$ and moment load capacities $M_{0}$ predicted by the $1 \mathrm{D}$ and 3DFE models. It is evident that the 1D model predictions agree very well with the 3DFE model predictions, with the largest difference being only $1.51 \%$ for the horizontal load capacity $H_{0}$.

Table 2. Comparison of the uniaxial global load capacities predicted by the 1D and 3DFE models

\begin{tabular}{lccc}
\hline Capacity & $1 \mathrm{D}$ & 3DFE & Diff $(\%)$ \\
\hline$V_{0} / A^{\text {base }} s_{\mathrm{u}}$ & 13.12 & 13.12 & 0.00 \\
$H_{0} / A^{\text {base }} s_{\mathrm{u}}$ & 6.01 & 5.93 & 1.51 \\
$M_{0} / A^{\text {base }} D s_{\mathrm{u}}$ & 3.7 & 3.7 & -0.07 \\
\hline
\end{tabular}

where $D$ is the caisson diameter and $A^{\text {base }}=\pi D^{2} / 4$

Figure 4 compares the global load-displacement predictions under uniaxial loading, where $w_{\mathrm{RP}}, u_{\mathrm{RP}}$ and $\theta_{\mathrm{RP}}$ are the vertical, horizontal and rotational displacements of the loading reference point RP. As observed, the 1D and 3DFE model predictions tend to reach the load capacity at different displacements.

Under pure vertical loading, the 1D model reaches load capacity at a smaller displacement than the 3DFE model. Moreover, it can be seen from the closeup inset that the 1D model load-displacement prediction is bilinear. The first linear response is the elastic response while the second linear response occurs when the base soil reaction has reached its local yield surface but the skirt soil reaction remains elastic.

Under pure horizontal loading, the 1D model predicts $u_{\mathrm{RP}} / D$ of 0.1 and $\theta_{\mathrm{RP}}$ of 0.129 , which compares well with the 3DFE model predictions of $u_{\mathrm{RP}} / D$ of 0.1 and $\theta_{\mathrm{RP}}$ of 0.139 . Similarly, under pure moment loading, the $1 \mathrm{D}$ model predicts $\theta_{\mathrm{RP}}$ of 0.1 and $u_{\mathrm{RP}} / D$ of 0.05 , which compares well with the 3DFE model predictions of $\theta_{\mathrm{RP}}$ of 0.1 and $u_{\mathrm{RP}} / D$ of 0.0556 .

Under pure horizontal or moment loading, the 1D model load-displacement predictions are not bilinear as both horizontal $h$ and rotational $m$ soil reactions occur during these loadings. The influence of combined $h$ and $m$ loading forces the soil reaction path to track on the local yield surface during elastoplastic yielding, until the global load capacity is reached.
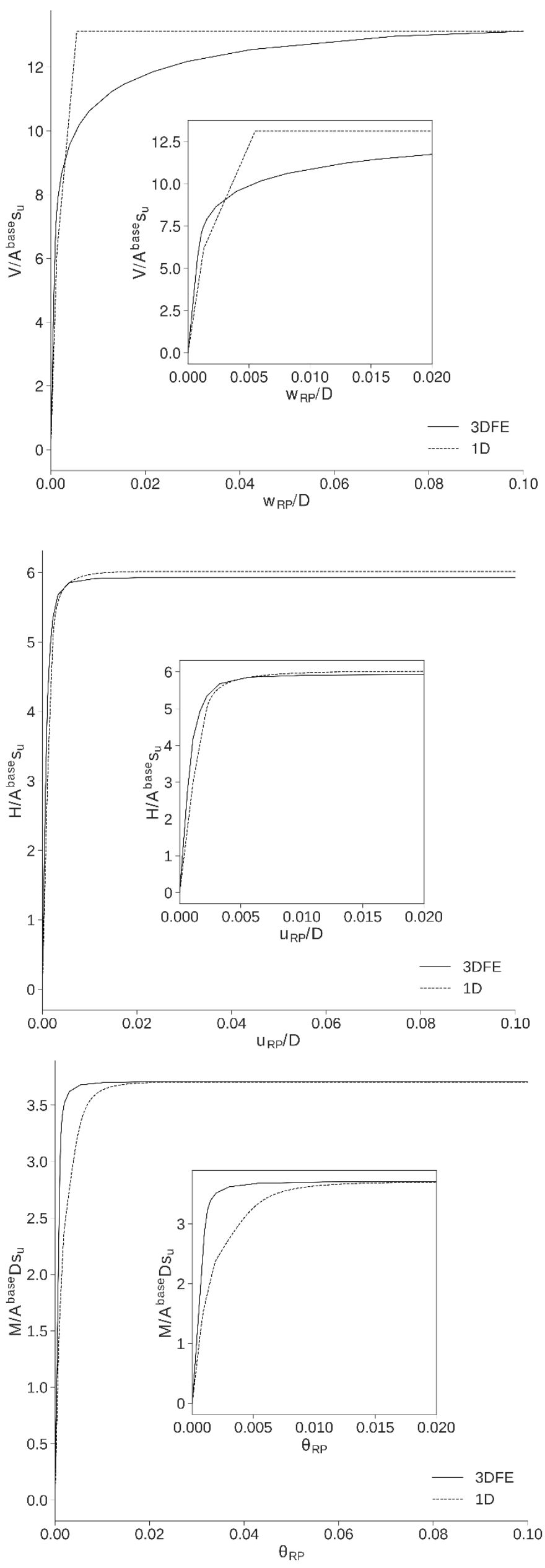

Figure 4. Comparison of global load-displacement predictions. The close-up insets focus on the results at small displacements. 


\subsection{Failure Envelopes}

Figure 5 compares the predictions of the $V H$ and $V M$ failure envelopes of the caisson in normalised forms, where the loads are normalised by their respective uniaxial capacities. The 1D model predictions of the $V H$ and $V M$ failure envelopes match the 3DFE results very well, albeit with a slight overprediction for the $\mathrm{VH}$ failure envelope for some load cases.
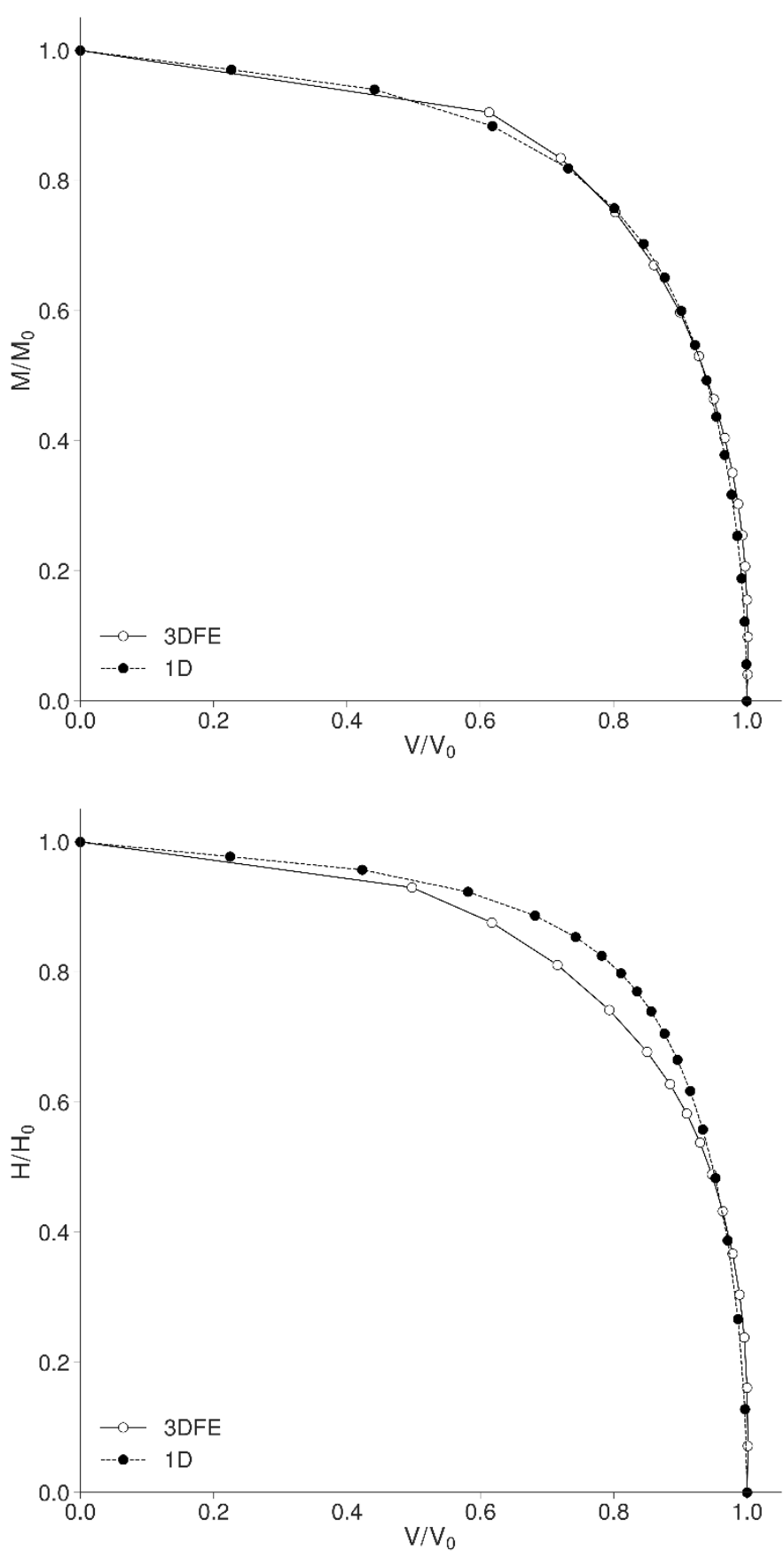

Figure 5. Comparison of global failure envelope predictions in the $V H$ and $V M$ load space.

Next, Figure 6 compares the predictions of the $V H M$ failure envelopes in normalised forms. Despite the poor match of the local yield surfaces at the local level (see Figure 3), the 1D model predictions of the global $H M$ envelope under fixed $V$ loads match the 3DFE predictions reasonably well.
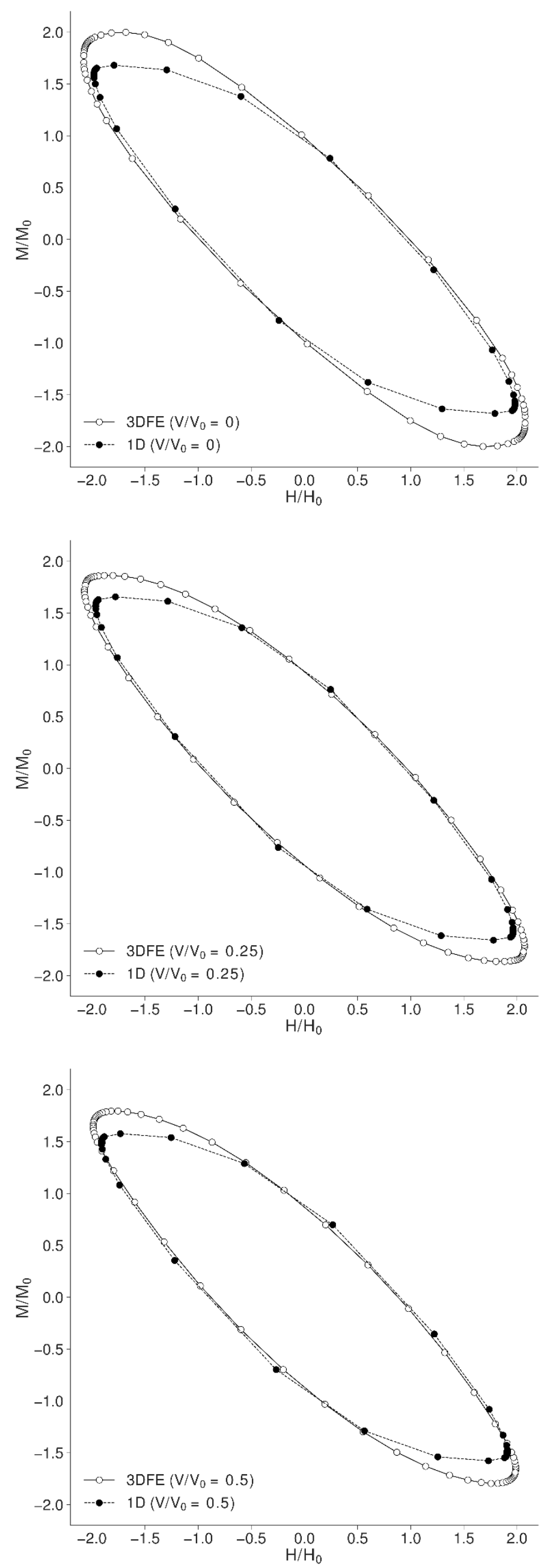


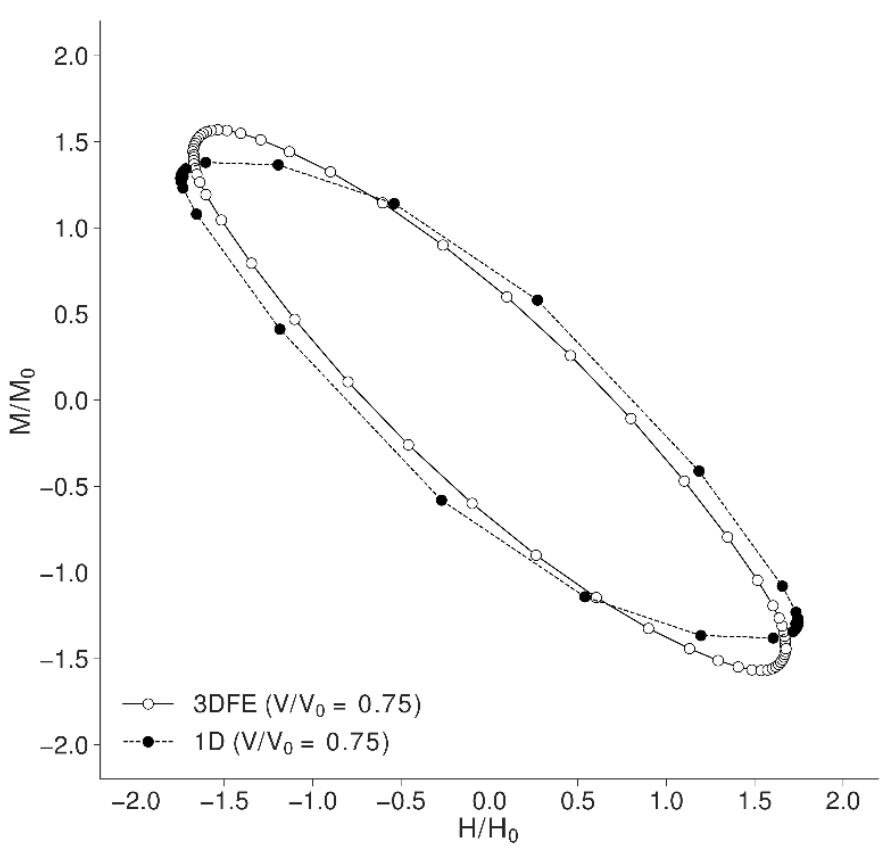

Figure 6. Comparison of global failure envelope predictions in the VHM load space.

Nevertheless, given the mismatch (especially that of the skirt local yield surface) in Figure 3, it is encouraging to see that the global failure envelope predictions are not too sensitive to the accuracy of these local yield surfaces. Furthermore, most loading scenarios are in the quadrants where $H$ and $M$ have the same sign. Thus, the mismatch in the quadrants where $H$ and $M$ have different signs are of less practical concern.

\subsection{Cyclic Loading}

To assess whether the 1D model can simulate hysteretic behavior, a single cycle of positive and negative vertical displacements $\left(w_{\mathrm{RP}} / \mathrm{D}= \pm 0.05\right)$ was prescribed onto the caisson. Figure 7 shows the comparison of the global load-displacement behavior under this cyclic loading. It is clear that the 1D model is able to simulate hysteresis, although the 1D model predictions is a rather crude piecewise linear approximation of the 3DFE model predictions.

By comparing the displacements at zero $V$ load, it is evident that the permanent displacement predictions of the 1D and 3DFE models are in good agreement. The 1D model comes with a built-in capability for simulating effects such as permanent displacement and hysteresis. This is not surprising as both the 1D and 3DFE models are based on fundamentally the same elastoplasticity concepts, but with different measures of 'stress' and 'strain'.

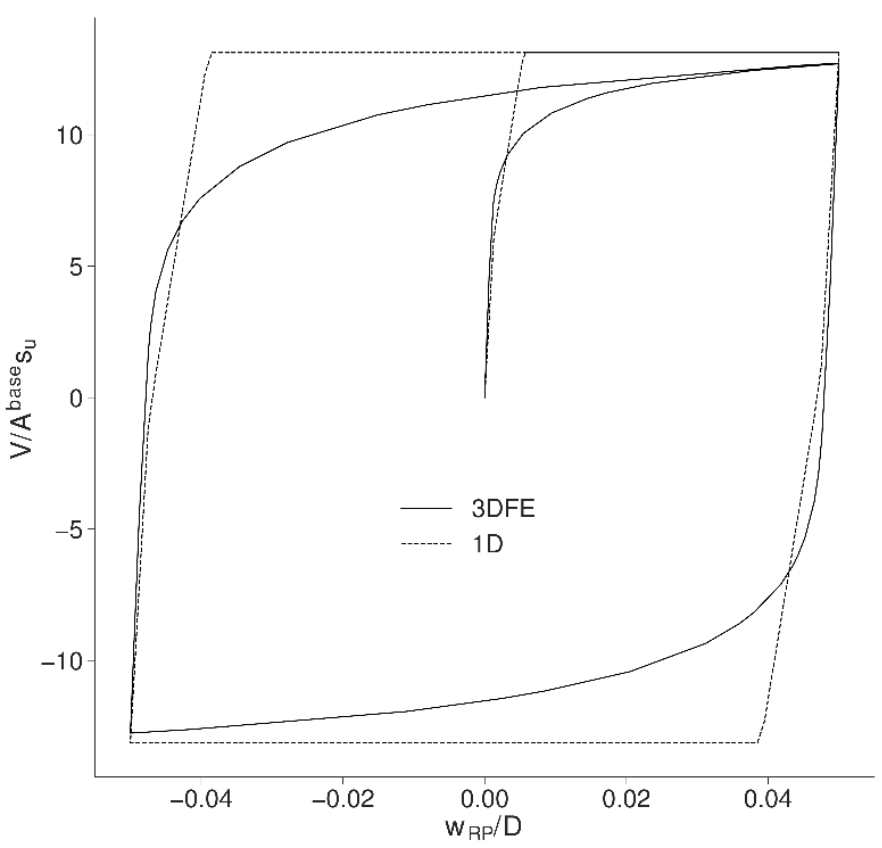

Figure 7. Comparison of global load-displacement behavior under cyclic vertical loading

\section{DISCUSSION}

The 1D model ignores much of the detail of the original 3D continuum-based problem, with the aim of appropriate simplification to provide a fast proxy to the original problem. Despite the simplifying abstractions, the loss in accuracy is minimal, relative to the large gains in computational efficiency. For example, the 3DFE model took about 28 hours in total to run the analyses presented in Section 3. By contrast, the 1D model took about 0.8 hours in total, yielding a time saving of $97 \%$.

This computational efficiency is very important for design optimization involving multiple foundations, such as that for an offshore wind farm. Whilst 3DFE is perhaps practical for design projects involving only a few foundations, it is clearly impractical when there are hundreds of foundations. A tool such as the trained 1D model offers the 3DFE accuracy but with much higher efficiency, and therefore allows more of the design space to be explored.

Furthermore, the proposed 1D model offers advantages over existing macro-element models for shallow foundations (e.g. Cassidy 2004, Salciarini et al. 2011). Given the localised nature of the soil reactions and the yield surfaces, the 1D model may be simply adapted to non-homogeneous or multi-layered grounds with arbitrary yield strength profiles. This contrasts with macro-element models, which can only be adapted to ground profiles similar to that in the original calibration. In other words, the 1D model is a more generalised model by comparison with the macro-element model.

The focus of this paper is a presentation of the mapping process from a 3DFE elastoplastic continuum model to a $1 \mathrm{D}$ elastoplastic Winkler model, and a 
demonstration of the accuracy of the approach. As such, generalised formulations of the yield surfaces, although established for caissons of $\mathrm{L} / \mathrm{D} \leq 2$, are not presented. They will be described in future publications.

There are, of course, some observed limitations with the 1D model. For example, the load predictions under purely vertical loading is bilinear. This could be resolved by adding multiple or nested local yield surfaces but this increase in accuracy comes at the expense of increased computational effort. Also, there is room for improvement for the global failure envelopes predicted by the 1D model and this can be achieved by adopting a more expressive function with more parameters to represent the local yield surface. However, while there are ready solutions to these limitations, it is advisable to consider whether the additional complexity balances the aim of providing a rapid but approximate solution to the caisson-soil interaction problem for preliminary designs, which can then be refined using more advanced 3DFE analyses.

\section{CONCLUSION}

The main concern with Winkler models that use nonlinear elastic soil reactions to approximate the soil continuum response is that they do not easily reproduce observed phenomena such as permanent displacement, hysteresis and influence of combined loading on failure states. This paper resolves this shortcoming by proposing a 1D Winkler model that couple linear elastic soil reactions with local plastic yield surfaces that limits the allowable soil reaction states. The results indicate that the proposed 1D model compares favourably with the 3DFE model predictions in terms of accuracy across a range of loading states. The principal advantage, however, is efficiency, as it takes only $3 \%$ of the computational time required by the 3DFE model. Furthermore, unlike macro-element models which can only be used for ground profiles that are similar to the original calibration, the 1D model can be used for non-homogeneous or multi-layered grounds with arbitrary yield strength profiles, making it a more general, and arguably, useful model. Thus, the proposed 1D model offers an efficient method to predict realistic, non-linear behavior of caissons in elastoplastic soil.

\section{REFERENCES}

API. 2010. RP 2A-WSD - Recommended Practice for Planning, Designing and Constructing Fixed Offshore Platforms. Washington: American Petroleum Institute

Bransby, M. F. \& Yun, G. J. 2009. The undrained capacity of skirted strip foundations under combined loading. Géotechnique, 59(2), pp.115-125.

Cassidy, M. J., Martin, C. M. \& Houlsby, G. T. 2004. Development and application of force resultant models describing jack-up foundation behaviour. Marine Structures, 17(3-4), pp.165-193.

DNV. 2014. OS-J101 - Design of Offshore Wind Turbine Structures. Oslo: Det Norske Veritas.

Dormand, J. R. \& P. J. Prince. 1980. A family of embedded Runge-Kutta formulae. Journal of Computational and Applied Mathematics, Vol. 6, pp.19-26.

Gerolymos, N. and Gazetas, G., 2006. Development of Winkler model for static and dynamic response of caisson foundations with soil and interface nonlinearities. Soil Dynamics and Earthquake Engineering, 26(5), pp.363-376.

Gourvenec, S. \& Barnett, S. 2011. Undrained failure envelope for skirted foundations under general loading. Géotechnique, 61(3), pp.263-270.

Salciarini, D., Bienen, B. \& Tamagnini, C. 2011. A hypoplastic macroelement for shallow foundations subject to six-dimensional loading paths. Proceedings of the 2 nd international symposium on computational geomechanics (COMGEO-II), USA.

Suryasentana, S.K., Byrne, B.W. Burd, H.J., \& Shonberg, A. 2017. Simplified Model for the Stiffness of Suction Caisson Foundations Under 6 DOF Loading. Proceedings of SUT OSIG 8th International Conference, London, UK.

Varun, Assimaki, D. and Gazetas, G., 2009. A simplified model for lateral response of large diameter caisson foundationsLinear elastic formulation. Soil Dynamics and Earthquake Engineering, 29(2), pp.268-291.

Vulpe, C., 2015. Design method for the undrained capacity of skirted circular foundations under combined loading: effect of deformable soil plug. Géotechnique, 65(8), pp.669-683. 